\title{
THE WEIGHTED VECTOR ALGORITHMS IN ONE PERSON AND ONE CRITERION
}

\author{
Qin Shoukang \\ Guangzhou Municipal Institute of Systems Engineering \\ Guangzhou Normal University, China \\ gise@guangztc.edu.cn
}

\begin{abstract}
The aim of this paper is to summarize the weighted vector algorithms in one person and one criterion of AHP. It includes three parts: The first is a sketch survey of the weighted vector algorithms in one person and one criterion. The second is to link up the relations in several algorithms, and to find the equivalence or the resemblance in them. The last is to study the common character of the optimization algorithms from methodology point of view.

Key Words one person and one criterion, the equivalence in algorithms, the resemblance in algorithms, disturbance function
\end{abstract}

\section{A sketch survey of the algorithms}

The weighted vector algorithms in one person and one criterion may be generally divided to three kinds: - eigenvector methods; - optimization methods; ' other methods.

Note generally in this paper $\mathrm{A}=\left[a_{i j}\right]_{n \times n}$ be judgement matrix, $\mathrm{A}^{*}=\left[w_{l} / w_{j}\right]_{n \times n}$ be weighted matrix to seek, $\mathrm{D}=\left\{\mathrm{w} \mid \sum_{i=1}^{n} w_{i}=1, w_{i}>0, i=1,2, \cdots, n\right\} \quad$ be weighted vector set, $\mathrm{e}=(1,1, \cdots, 1)^{T} \in R_{+}^{n}$.

\subsection{Eigenvector methods}

Eigenvector methods are basic algorithms of AHP.

There is a simply algorithm * power method for finding eigenvector when $\mathrm{A}>0$.

If $\mathrm{A}>0, \mathrm{x}=\mathrm{e}$, then

$$
\lim _{k \rightarrow \infty} \frac{\mathrm{A}^{k} \mathrm{x}}{\mathrm{X}^{T} \mathrm{~A}^{k} \mathrm{X}}=\mathrm{w}
$$

where $\mathrm{W}$ is normal eigenvector corresponding to maximum eigenvalue of $\mathrm{A}$, named weighted vector or sequence vector.

The algorithm of power method is as follows: Given accuracy $\varepsilon>0$.

(1) Take $\mathrm{x}^{(0)}=\mathrm{e}$, let $k=0$.

(2) Calculate $\mathrm{x}^{(k+1)}=\mathrm{Ax} \mathrm{x}^{(k)}=\mathrm{A}^{(k+1)} \mathrm{e}$.

(3) Let $\beta_{k+1}=\mathrm{e}^{T} \mathrm{x}^{(k+1)}=\sum_{i=1}^{n} x_{i}(k+1)$, Calculate normal vector $\mathrm{y}^{(k+1)}=\mathrm{x}^{(k+1)} / \beta_{k+1}$.

(4) If

$$
\left|y_{i}^{(k+1)}-y_{i}^{(k)}\right|<\varepsilon \quad i=1,2, \cdots, n
$$

hold, then weighted vector $\mathrm{w}=\mathrm{y}^{(k+1)}$, turn to (5); otherwise let $k=k+1$, return to (2). 


$$
c_{i j}=\frac{1}{n} \sum_{k=1}^{n} \lg \left(\frac{a_{i k}}{a_{j k}}\right)
$$

also let $a_{i j}^{*}=10^{c_{j y}}$, construct quasi-optimal compatibility matrix $\hat{\mathrm{A}}=\left[\mathrm{a}_{i j}^{*}\right]_{n \times n}$. According to the following formula

(5) Calculate maximum eigenvalue of $\mathrm{A}$

$$
\lambda_{\max }=\sum_{i=1}^{n}(\mathrm{Aw})_{i} / n w_{i}
$$

\subsection{Optimization methods}

Optimization methods may be divided to logarithmic least square method, least square method, least deviation method and $\chi^{2}$ method etc. according to the denoted manner of disturbance matrices.

\subsubsection{Logarithmic least square method (LLSM)}

Define disturbance matrix $\mathrm{E}=\left[\varepsilon_{i j}\right]_{n \times n}=\mathrm{A} \circ \mathrm{A}^{* T}=\left[a_{i j} \frac{w_{j}}{w_{l}}\right]$, where " $\circ$ " is Hadamard product. Construct logarithmic least square model:

$$
\min f(\mathrm{w})=\sum_{i, j=1}^{n} \lg ^{2} \varepsilon_{i j}=\sum_{i, j=1}^{n}\left[\lg \left(a_{i j} \frac{w_{j}}{w_{i}}\right)\right]^{2}
$$

through simple calculation, we obtain

$$
\mathrm{W} \in \mathrm{D}
$$

$$
w_{i}=\frac{\left(\prod_{j=1}^{n} a_{i j}\right)^{\frac{1}{n}}}{\sum_{k=1}^{n}\left(\prod_{j=1}^{n} a_{k j}\right)^{\frac{1}{n}}} \quad i=1,2, \cdots, n
$$

Obviously, above result is the same as the result from root method.

In [2]、 [3], applied the concept of optimal transmission matrix, translate the elements in judgement matrix $\mathrm{A}=\left[a_{i j}\right]_{n \times n}$ into

calculate weights.

$$
w_{j}=\frac{1}{\sum_{i=1}^{n} a_{i j}^{*}} j=1,2, \cdots, n
$$

In [4], defined $a_{i j}$ in $\mathrm{A}=\left[a_{i j}\right]_{n \times n}$ be direct judge element for thing $i$ to thing $j, a_{i k} / a_{j k}$ $(k=1,2, \cdots n)$ be indirect judge element for thing $i$ to thing $j$, and take geometric mean of the latter

construct compatibility matrix

$$
a_{i j}^{*}=\left(\prod_{k=1}^{n} \frac{a_{i k}}{a_{j k}}\right)^{\frac{1}{n}}
$$

from this obtain weights

$$
\hat{\mathrm{A}}=\left[\mathrm{a}_{i j}^{*}\right]
$$




$$
w_{j}=\frac{1}{\sum_{i=1}^{n} a_{i j}^{*}} \quad j=1,2, \cdots, n
$$

\subsubsection{Least square method (LSM),}

Define disturbance matrix $\mathrm{E}=\left[\varepsilon_{i j}\right]_{n \times n}=\mathrm{A}-\mathrm{A}^{*}=\left[a_{i j}-w_{i} / w_{j}\right]$. Construct least square model:

$$
\begin{gathered}
\min g(\mathrm{w})=\sum_{i, j=1}^{n}\left(a_{i j}-w_{i} / w_{j}\right)^{2} \\
\mathrm{w} \in \mathrm{D}
\end{gathered}
$$

There are some LSM in references [5], [6] and [7].

In [6], constructed typical quadratic form problem:

$$
\begin{aligned}
\min J & =\mathrm{z}^{T} \mathrm{z} \\
\text { s.t. Aw } & =n \mathrm{w}+\mathrm{z} \\
\mathrm{e}^{T} w & =1 \\
w & >0
\end{aligned}
$$

its solution is

$$
\mathrm{w}=\frac{\operatorname{Re}}{\mathrm{e}^{T} \mathrm{Re}}
$$

where

$$
\mathrm{R}=\left[(\mathrm{A}-n \mathrm{I})^{T}(\mathrm{~A}-n \mathrm{I})\right]^{-1}
$$

\subsubsection{Least deviation method(LDM)}

Define disturbance matrix $\mathrm{E}=\left[\varepsilon_{i j}\right]_{n \times n}=\left[a_{i j} \frac{w_{j}}{w_{i}}\right]$, and construct least deviation model:

$$
\min h(\mathrm{w})=\frac{1}{2} \sum_{i, j=1}^{n}\left(a_{i j} \frac{w_{j}}{w_{i}}+a_{j i} \frac{w_{i}}{w_{j}}-2\right)
$$

[8] gave following theorem and algorithm:

$$
\mathrm{w} \in \mathrm{D}
$$

Theorem 1 Function $h(\mathrm{w})$ exists unique minimum solution $\mathrm{w}^{*} \in \mathrm{D}$, which also is the only solution on $\mathrm{D}$ of equations

$$
\sum_{j=1}^{n} a_{i j} \frac{w_{j}}{w_{i}}-\sum_{j=1}^{n} a_{j i} \frac{w_{i}}{w_{j}}=0 \quad i=1,2, \cdots, n
$$

The algorithm of least deviation method is as follows: Note

$$
\varphi_{i}(\mathrm{w}(k))=\sum_{j=1}^{n} a_{i j} \frac{w_{j}(k)}{w_{i}(k)}-\sum_{j=1}^{n} a_{j i} \frac{w_{i}(k)}{w_{j}(k)} \quad i=1,2, \cdots, n
$$

Given accuracy $\varepsilon>0$, select initial weighted vector $\mathrm{w}(0)=\left\{w_{1}(0), w_{2}(0), \cdots, w_{n}(0)\right\} \in \mathrm{D}$ and let $k=0$.

(1) Calculate $\varphi_{i}(\mathrm{w}(k)), i=1,2, \cdots, n$. If $\left|\varphi_{i}(\mathrm{w}(k))\right|<\varepsilon$ hold for $\forall i$, then $\mathrm{w}(k)$ is minimum solution of $h(\mathrm{w})$, stop; otherwise turn to (2).

(2) Note 


$$
\left|\varphi_{l}(\mathrm{w}(k))\right|=\max _{i}\left|\varphi_{i}(\mathrm{w}(k))\right|
$$

find

$$
\begin{gathered}
t(k)=\left(\sum_{j \neq l} a_{l j} \frac{w_{j}(k)}{w_{l}(k)} / \sum_{j \neq l} a_{j l} \frac{w_{l}(k)}{w_{j}(k)}\right)^{\frac{1}{2}} \\
x_{i}(k)= \begin{cases}t(k) w_{l}(k), & i=l \\
w_{i}(k), & i \neq l\end{cases} \\
w_{i}(k+1)=x_{i}(k) / \sum_{j=1}^{n} x_{j}(k)
\end{gathered}
$$

let $k=k+1$, return to (1).

\subsection{4 $\chi^{2}$ method $^{[9]}$}

Define disturbance matrix $\mathrm{E}=\left[\varepsilon_{i j}\right]_{n \times n}=\left[a_{i j} \frac{w_{j}}{w_{i}}\right]$ and construct $\chi^{2}$ model:

$$
\min r(\mathrm{w})=\sum_{\substack{i, j=1 \\ \mathrm{w} \in \mathrm{D}}}^{n} \frac{\left(a_{i j}-w_{i} / w_{j}\right)^{2}}{w_{i} / w_{j}}
$$

The algorithm of $\chi^{2}$ method is as follows: Note

$$
\varphi_{i}(\mathrm{w}(k))=\sum_{j=1}^{n}\left[\left(1+a_{i j}^{2}\right) \frac{w_{j}(k)}{w_{i}(k)}-\left(1+a_{j i}^{2}\right) \frac{w_{i}(k)}{w_{j}(k)}\right] \quad i=1,2, \cdots, n
$$

Given accuracy $\varepsilon>0$, select initial weighted vector $\mathrm{w}(0)=\left\{w_{1}(0), w_{2}(0), \cdots, w_{n}(0)\right\}^{T} \in \mathrm{D}$ and let $k=0$.

(1) Calculate $\varphi_{i}(\mathrm{w}(k)), i=1,2, \cdots, n$. If $\left|\varphi_{i}(\mathrm{w}(k))\right|<\varepsilon$ hold for $\forall i$, then $\mathrm{w}(k)$ is minimum solution of $r(\mathrm{w})$, stop; otherwise turn to (2).

(2) Note

$$
\left|\varphi_{l}(\mathrm{w}(k))\right|=\max _{i}\left|\varphi_{i}(\mathrm{w}(k))\right|
$$

find

$$
\begin{aligned}
& t(k)=\left[\sum_{j \neq l}\left(1+a_{l j}^{2}\right) \frac{w_{j}(k)}{w_{l}(k)} / \sum_{j \neq l}\left(1+a_{j l}^{2}\right) \frac{w_{l}(k)}{w_{j}(k)}\right]^{\frac{1}{2}} \\
& x_{l}(k)= \begin{cases}t(k) w_{l}(k), & i=l \\
w_{i}(k), & i \neq l\end{cases} \\
& w_{i}(k+1)=x_{i}(k) / \sum_{j=1}^{n} x_{j}(k) \quad i=1,2, \cdots, n
\end{aligned}
$$

let $k=k+1$, return to (1).

\subsection{Other methods} latter.

They are gradient eigenvector method and relative entropy method. Now describe the result of the

[10] Constructed following relative entropy model: 


$$
\min q(\mathrm{w})=\sum_{i, j=1}^{n}\left(\lg w_{i}-\lg \frac{a_{i j}}{\sum_{k=1}^{n} a_{k j}}\right) w_{i}
$$

Applying Lagrange multiplier method, we obtain

$$
w_{i}=\prod_{j=1}^{n}\left(\frac{a_{i j}}{\sum_{k=1}^{n} a_{k j}}\right)^{\frac{1}{n}} / \sum_{i=1}^{n} \prod_{j=1}^{n}\left(\frac{a_{i j}}{\sum_{k=1}^{n} a_{k j}}\right)^{\frac{1}{n}}, \quad i=1,2, \cdots, n
$$

\section{The equivalence and the resemblance in algorithms}

Two algorithms are said to be equivalence, if the calculation formulae of weighted vectors in both are the same. Two algorithms are said to be resemblance, if the theory and methods for finding weighted vectors in both are duplicate.

\subsection{The equivalence in algorithms}

The following several algorithms are equivalence:

1) Applied simple logarithm knowledge, easy verify that the result in [2], [3] is the same as the result in [4], and may be denoted to expression $\cdot$. It implies that they are equivalence with LLSM.

2) If let $k=1$ in formula $\cdot$, we obtain

$$
w_{i}=\frac{(\mathrm{Ae})_{i}}{\mathrm{e}^{T} \mathrm{Ae}}=\frac{\sum_{j=1}^{n} a_{i j}}{\sum_{k=1}^{n} \sum_{j=1}^{n} a_{k j}}, \quad i=1,2, \cdots, n
$$

It is just the expression of sum method. It implies that sum method is equivalence with approximation of 1st degree of power method.

3) The expression - may be simplified to

$$
w_{i}=\prod_{j=1}^{n}\left(a_{i j}\right)^{\frac{1}{n}} / \sum_{k=1}^{n} \prod_{j=1}^{n}\left(a_{k j}\right)^{\frac{1}{n}}, \quad i=1,2, \cdots, n
$$

It is the same as formula $\cdot$ Therefore, the relative entropy method is equivalence with LLSM.

\subsection{The resemblance in algorithms}

Expanding expression $\cdot$, we obtain

$$
\begin{aligned}
r(\mathrm{w}) & =\sum_{i, j=1}^{n} \frac{\left(a_{i j}-w_{i} / w_{j}\right)^{2}}{w_{i} / w_{j}} \\
& =\sum_{i, j=1}^{n} a_{i j}\left(a_{i j} \frac{w_{j}}{w_{i}}+a_{j i} \frac{w_{i}}{w_{j}}-2\right)
\end{aligned}
$$

Obviously, as function of $\mathrm{w}$, the expressions in parentheses of $h(\mathrm{w})$ and $r(\mathrm{w})$ are the same. It implies that these algorithms must have their parallel results in theory and method. 


\section{Some conclusions}

1) The common properties in optimization methods

Four optimization methods * LLSM, LSM, LDM and $\chi^{2}$ method have following two common properties:

(1) The construction forms of goal functions are decided by the expressions of disturbance functions. The disturbance functions can be induced two basic types: (i) ratio type: $\varepsilon_{i j}=a_{i j} /\left(\frac{w_{i}}{w_{j}}\right)$, its ideal value is $\varepsilon_{i j}=1$; (ii) difference type: $\varepsilon_{i j}=a_{i j}-\frac{w_{i}}{w_{j}}$, its ideal value is $\varepsilon_{i j}=0$, and the ideal values of both are arrived when the judgement matrices are compatible.

(2) In four models $\cdot, \cdot \cdot, \cdot, w \in D \subset R_{+}^{n}, D$ are open sets. Therefore, their necessary conditions can be found with Lagrange multiplier method.

2) The weighted vector algorithms in several persons and one criterion are generalized from the weighted vector algorithms in one person and one criterion. Therefore, there are similar conclusions on equivalence and resemblance in algorithms, see [11] in detail.

\section{References}

[1] 王莲芬、许树柏，(1990）层次分析法引论, 中国人民大学出版社。

[2] 梁梁等, (1989) “一种改进的层次分析法”, 系统工程, 31, 5一7.

[3] 梁梁等, (1991) “对 “一种改进的 AHP 法” 的补注”, 系统工程, 43, 64-65。

[4] 王应明等, (1993) “判断矩阵一致性逼近的一种新方法”, 决策与决策支持水统, 4, $79-84$

[5] Saaty, T.L. and Vargas, L.G. (1984) "Comparison of eigenvalue, logarithmic least squares and least squares methods in estimating rations" , Mathematical Modeling, 5.

[6] 王应明等, (1991) “优化理论在层次分析法中的应用”, 系统工程理论与实践, 2, 24 $-29$.

[7] 王应明等, (1995) “关于层次分析法中权的最小平方法的理论证明”, 系统工程理论 与实践, $1,3-8$.

[8] 陈宝谦, (1990) “层次分析的两种新排序方法”, 系统工程学报, 2, 43-51.

[9] 王应明等, (1992) “一种用于群组判断矩阵排序的 $\chi^{2}$ 方法”, 决策与决策支持系统, 3. 48-55.

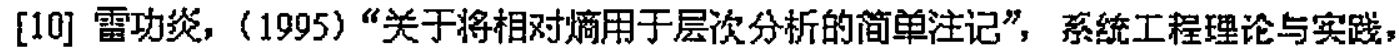
3 , 65-68.

[11] 素寿康, (1998) “多人层次单排序下权重向量的算法”, 系统工程理论与实践, 7, $89-93$. 